\title{
Taking in Account Measuring Errors of Volume Conversion Devices in Measuring of the Volume of Natural Gas
}

\author{
Franc Cimerman ${ }^{1,}$ - Matej Jarm 1 - Branko Širok² - Bogdan Blagojevič ${ }^{1}$ \\ 1 Plinovodi d.o.o., Slovenia \\ 2 University of Ljubljana, Faculty of Mechanical Engineering, Slovenia
}

In order to measure standard volumes of natural gas we use volume-conversion devices (VCDs) or correctors. The European standard EN 12405-1 specifies the requirements and tests for the construction, performance, safety and conformity of VCDs associated with gas meters. VCDs are used to convert measurement flow, temperature, pressure and gas composition under operating conditions into base conditions. Gas-volume conversion devices consist of a calculator and a temperature transducer, or a calculator, a temperature transducer and a pressure transducer that are locally installed. On the basis of this standard we separate VCDs into type 1 and type 2. Type 1 VCDs are complete instruments, while type 2 VCDs are made of separate elements. The measuring errors for type 1 VCDs are determined for three different temperature ranges and for five different pressures. With the intention of determining the detected quantity of natural gas as accurately as possible, this article introduces the possibility of using measurement errors that are obtained by the national verification procedure. With the European MID (Measuring Instrument Directive 2004/22/EC) the maximum permissible error for type 1 and type 2 VCDs is $\pm 0.5 \%$. Using the suggested methods of implementing measurement errors in this article, we can meet the MID requirements for any type 1 and type 2 VCDs, the estimated measurement uncertainty, in addition to the $1 / 3$ tolerable measuring errors, also takes into account the measurement uncertainty from the approximation of the proposed method.

For different electronic natural-gas VCDs we wish to approximate the measurement errors that are determined during their testing using the regression method of least squares and determine the model's characteristics.

On the basis of a data analysis this article proposes statistical measures for determining the suitability of the regression model for a particular VCD. This article also proposes a verification procedure for taking into account experimental design theory, regression analysis, and a reduced number of measurement points. The proposed method is suitable for both types of VCDs.

Keywords: volume conversion devices (correctors), measuring procedure, multiregression, measuring uncertainty

Highlights

- $\quad$ The measuring error of the volume of natural gas can be reduced by taking in account measuring error of VCD and gas meter.

- The important statistical estimators for estimating the suitability of a multi-regression analysis, used for any natural-gas VCD, are the standard error of the estimate SEE, the regression coefficient $r^{2}$ and the significance $F$ respectively.

- The proposed method of measurement can represent an upgrade to the national verification procedures for the testing of VCDs.

- A reduced number of measurement points is proposed for the internal testing of the VCDs in the field.

\section{INTRODUCTION}

Electronic volume-conversion devices (VCDs) or correctors are used to measure standard volumes of natural gas. VCDs are used to convert the measurement flow, temperature, pressure and gas composition under operating conditions into base conditions. The testing and verification of VCDs take into account some specifies and requirements of the standard EN 12405-1 [1] or the recommendation of PTB [2]. National authorities may decide to implement maximum-permissible errors for subsequent or in-service verification. The meter authentication specifies [3] and directive MID [4].

Systems for measuring standard gas volumes (Fig. 1) are comprised of a VCD and a gas meter. Gasvolume conversion devices consist of a calculator and a temperature transducer, or a calculator, a temperature transducer and a pressure transducer that are locally installed. The conversion as a function of temperature, is just called $t$-conversion, the conversion as a function of the pressure and of the temperature with a constant compression factor, is called $p t$-conversion, and the conversion as a function of the pressure, the temperature and taking into account the compression factor, is called $p t Z$-conversion. On the basis of the EN 12405-1 [1] we separate VCDs into type 1 and type 2. Type 1 VCDs are complete instruments, while type 2 VCDs are made of separate elements. Standard volumes of natural gas are measured at the temperature $T_{s}=288.15 \mathrm{~K}$ and pressure $p_{s}=1.01325$ bar. The VCD calculates and converts the volume of natural gas $V$, measured by the gas meter at pressure $p$, and absolute temperature $T$, into the volume under standard or norm conditions. The VCD's correction factor $C$ is defined on the basis of the standard EN 12405-1 [1] with: 


$$
V_{s}=\left(\frac{T_{s} \cdot p \cdot Z_{s}}{T \cdot p_{s} \cdot Z}\right) \cdot V=C \cdot V,
$$

where $Z$ is the compressibility factor of the natural gas, $p$ is its absolute pressure, and $T$ is its absolute temperature. From Eq. (1) we can see that the expression in brackets is equal to the variable $C$, which is the correction factor of the corrector:

$$
C=\left(\frac{T_{s} \cdot p \cdot Z_{s}}{T \cdot p_{s} \cdot Z}\right)=\left(\frac{T_{s} \cdot p}{T \cdot p_{s}} \frac{1}{K}\right),
$$

where $K$ is the compressibility of natural gas.

The thermodynamic transformations and characteristics of ideal as well as real gases, of which natural gas is one, are well described in [5]. The compressibility of natural gas is calculated on the basis of its known molar composition with the use of the algorithm AGA-DC92, or with the algorithm S-GERG88, where the calorific value superior $H_{s}$, the relative density $d$, and the molar fractions of carbon dioxide $x_{\mathrm{CO} 2}$ and hydrogen $x_{\mathrm{H} 2}$ must be known. Both algorithms are described in the standard EN 12213 [6]. The influence of the reference condition correction on a natural-gas flow measurement is well described in [7]. An experimental analysis was carried out on the Italian distribution system.

In the paper [8] the authors present a detailed uncertainty analysis of energy measurements for natural gas. The energy-measurement system, in an indirect way, consists of a gas-flow meter, a volumeconversion device and a gas chromatograph or gas analyser. The natural-gas measurements and billing are performed using volume measurements.
In the paper [9] the authors investigate the unaccounted for gas (UAG) sources and evaluate the criticalities related to UAG estimation-predictions. Inaccurate measurements can be responsible for a large UAG with significant financial losses.

The testing of natural-gas VCDs is carried out in accredited laboratories that must have a measurement uncertainty at least three times lower than the maximum-permissible errors (MPEs) [2], [3] and [10]. The measurement of the calorific value superior and its effect on the measure of uncertainty of the transferred quantities of natural gas are also described in [11].

\section{THEORETHICAL BACKGROUND}

With the intention of measuring an unknown volume of natural gas with the highest possible precision under standard or norm conditions, we must take in account the measurement errors of gas meters and VCDs that were defined during the testing. Fig. 2 demonstrates the mass flow rate of the gas $q_{m}$ through a pipeline, where two gas meters with their corresponding VCDs are installed in close succession.

At the first measuring point, marked with the index 1 , the measuring system detects the standard volume $V_{S 1}$; at the second measuring point, marked with the index 2, it detects the volume $V_{S 2}$. Each meter has its own measurement error. Hence, both gas meters have their corresponding errors $f_{V 1}$ and $f_{V 2}$. In addition, both VCDs have their own errors, namely, $f_{C 1}$ and $f_{C 2}$. Because both measuring points are influenced by the measuring errors, the ideal standard volume is equal to:

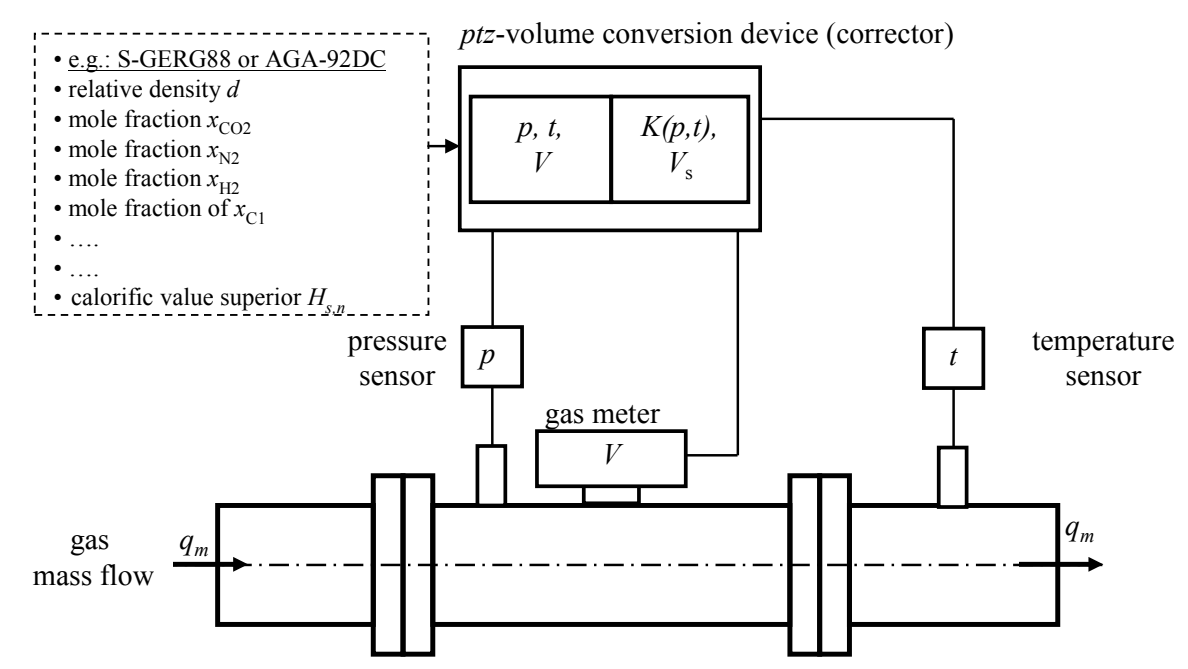

Fig. 1. Measurement system schematic for measuring standard volumes of natural gas 


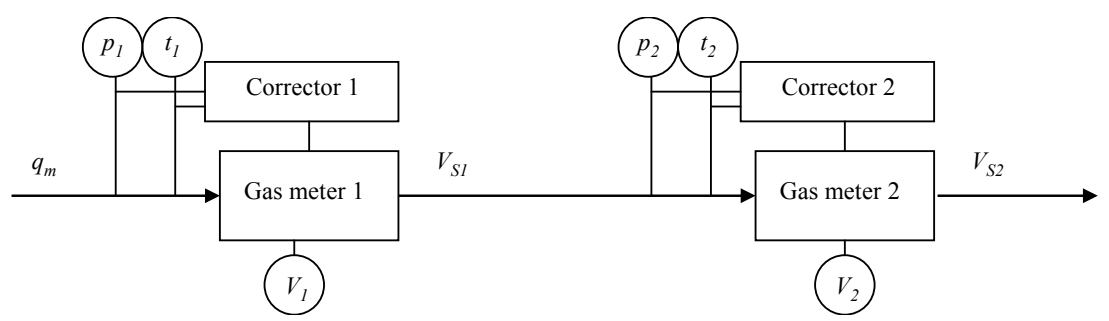

Fig. 2. Comparative measuring of standard volume of natural gas

$$
\begin{aligned}
V_{\text {Sid }} & =\frac{V_{1}}{1+f_{V 1}} \frac{C_{1}}{1+f_{C 1}}=\frac{V_{2}}{1+f_{V 2}} \frac{C_{2}}{1+f_{C 2}}= \\
& =\frac{V_{S 1}}{\left(1+f_{V 1}\right)\left(1+f_{C 1}\right)}=\frac{V_{S 2}}{\left(1+f_{V 2}\right)\left(1+f_{C 2}\right)} .
\end{aligned}
$$

The measurement errors $f_{V}$ and $f_{C}$ can only be determined by testing in laboratory conditions.

\subsection{Taking into Account the Measurement Errors of the Gas Meters}

The measurement error of the gas meter $f_{V}$ is determined on the basis of calibrating the gas meter with natural gas at a set operating pressure. On the basis of the [12] it is suggested, for turbine gas meters, to use the following approximation formula that well describes measurement errors of the gas meter:

$$
f_{V}=\mathrm{a}_{0}+\mathrm{a}_{1} x+\mathrm{a}_{2} x^{2}+\mathrm{a}_{3} x^{3}+\mathrm{a}_{4} x^{4}+\mathrm{a}_{\mathrm{T}}\left(293.15-T_{N}\right)
$$

where the quantity $x$ is $x=\log \left(R e_{N} / 106\right), T_{N}$ is the absolute temperature of the standard and $a_{i}$ are the coefficients of the approximation formula. The Reynolds number $R e_{N}$ is defined with the following expression:

$$
\operatorname{Re}_{N}=\frac{w \cdot D}{v}=\frac{4 \cdot q_{V}}{\pi D} \frac{\rho}{\eta} .
$$

Here, $w$ is the velocity of the gas, $D$ is the hydraulic diameter of the pipe, $v$ is the kinematic viscosity of the gas, $\eta$ is the dynamic viscosity of the gas, $\rho$ is the density of the gas, and $q_{V}$ is the volume flow rate of the gas.

The measurement errors of the gas meters for different flow rates, measured at a set pressure $p$, can be entered into flow computers or correctors. Using this method we can reduce the influence of the gas meter, and consider only the measuring uncertainty of the testing facility, which, as per MID, must not exceed $1 / 3$ of the allowable measurement error, which means at most $0.3 \%$ [2] to [4].

\subsection{Taking into account the measurement errors of the vCDs}

During the testing of the natural-gas VCDs we tested their metrological properties. For this reason we require a testing facility that is comprised of [1] and [2] to test the natural-gas VCDs:

The testing facilities measuring the uncertainty for the testing of electronic natural-gas volume VCDs, as shown in Fig. 3, must be lower than one-third of the maximum permissible errors (MPEs) for VCDs. The MPEs are listed in Table 1.

Table 1. The maximum-permissible errors (MPEs) on the basis of the MID specifications and/or Rules on measuring instruments for corrector testing [1] to [4] and [8]

\begin{tabular}{clll}
\hline $\begin{array}{c}\text { vCD } \\
\text { (corr.) }\end{array}$ & \multicolumn{1}{c}{ Conditions } & \multicolumn{1}{c}{$\begin{array}{c}\text { For reference } \\
\text { conditions } \\
{[\%]}\end{array}$} & $\begin{array}{c}\text { For rated } \\
\text { operating } \\
\text { conditions [\%] }\end{array}$ \\
\hline \multirow{2}{*}{ Type 1} & $\begin{array}{l}\text { Main indication } \\
(p, T, Z \text { and } p, T), f_{C}\end{array}$ & \pm 0.5 & \pm 1.0 \\
\cline { 2 - 4 } & Main indication (only $T), f_{C}$ & \pm 0.5 & \pm 0.7 \\
\hline \multirow{3}{*}{ Mype 2} & $\begin{array}{l}\text { Main indication } \\
(p, T, Z \text { and } p, T), f_{C}\end{array}$ & \pm 0.5 \\
\cline { 2 - 4 } & Calculator unit, $f_{\text {Calc }}$ & \pm 0.2 & \pm 0.3 \\
\cline { 2 - 4 } & Temperature sensor, $f_{T}$ & \pm 0.1 & \pm 0.2 \\
\cline { 2 - 4 } & Pressure sensor, $f_{T}$ & \pm 0.2 & \pm 0.5 \\
\cline { 2 - 4 } & Main indication $($ only $T), f_{C}$ & \pm 0.5 & \pm 0.7 \\
\hline
\end{tabular}

A VCD test is comprised of [1] and [2]:

- external inspection: with an external inspection it is possible to test whether the VCD has an official marking, which was issued with a certificate of approval. It is also checked to see whether the VCD has any significant external damage;

- inspection of the input and output constants: the adjustment of the input parameters and the impulse constants is inspected $\left[\mathrm{imp} / \mathrm{m}^{3}\right]$;

- procedures for determining the measurement errors: relative measurement errors for the type $1 \mathrm{VCDs}$ are determined at three different 


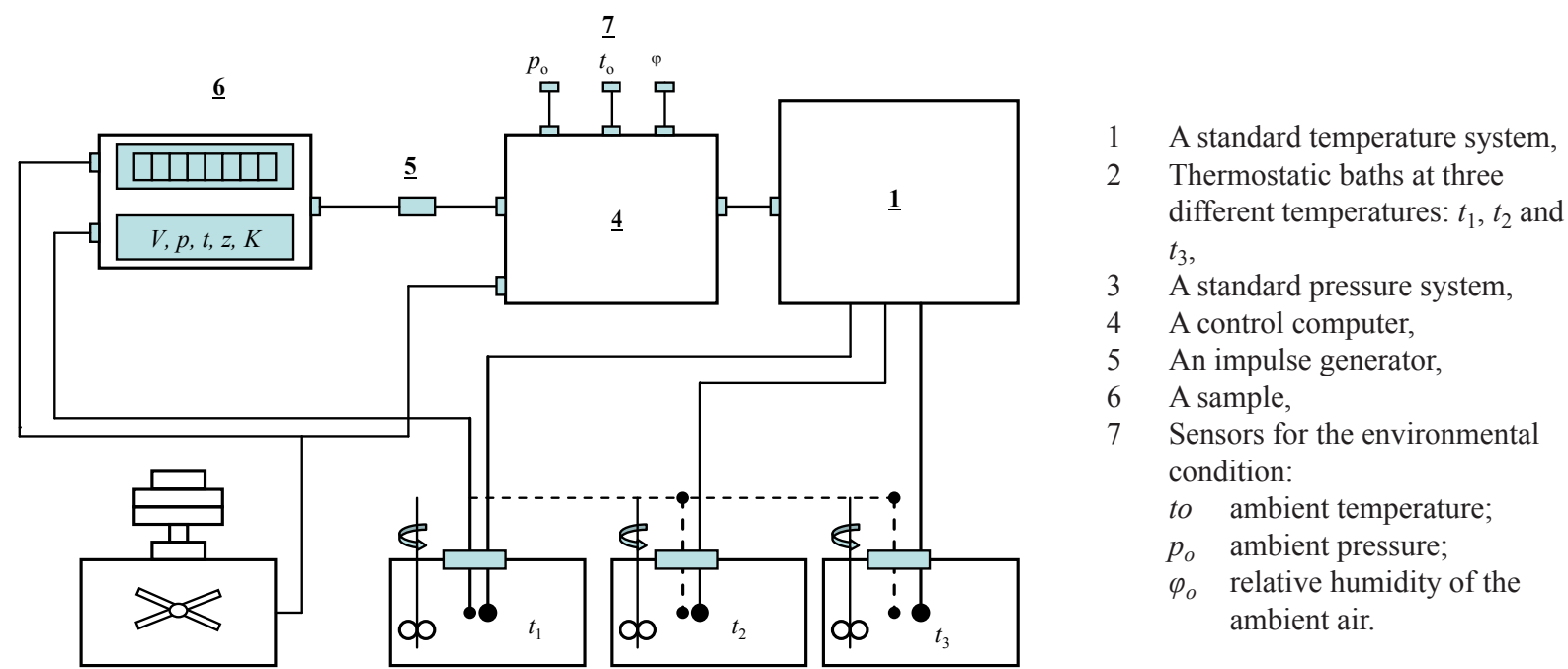

$\underline{\mathbf{3}}$

$\underline{2}$

Fig. 3. Testing facility schematic for VCD testing

temperatures and five different pressures for each temperature. The order of the determined points is as listed in Table 2 [1].

Table 2. Measuring points for type 1 VCD testing [1]

\begin{tabular}{cccccc}
\hline & $p_{1, \min }$ & $p_{2}$ & $p_{3}$ & $p_{4}$ & $p_{5, \max }$ \\
\hline$t_{1}\left[{ }^{\circ} \mathrm{C}\right]$ & $1 \rightarrow$ & $2 \rightarrow$ & $3 \rightarrow$ & $4 \rightarrow$ & $5 \downarrow$ \\
\hline$t_{2}\left[{ }^{\circ} \mathrm{C}\right]$ & $10 \downarrow$ & $9 \leftarrow$ & $8 \leftarrow$ & $7 \leftarrow$ & $6 \leftarrow$ \\
\hline$t_{3}\left[{ }^{\circ} \mathrm{C}\right]$ & $11 \rightarrow$ & $12 \rightarrow$ & $13 \rightarrow$ & $14 \rightarrow$ & 15 \\
\hline
\end{tabular}

We begin the test at the lowest temperature $t_{1}$. At this temperature we conduct tests for all five pressures, from $p_{1}=p_{\min }$ to $p_{5}=p_{\max }$. Then, at the next temperature, $t_{2}$, we measure the points in reverse order, from $p_{5}$ to $p_{1}$. The procedure for temperature $t_{3}$ is the same as for $t_{1}$. The test must be carried out at three different temperatures $t_{1}, t_{2}$, and $t_{3}$, where [1]:

$$
t_{2}=\frac{t_{1}+t_{3}}{2} .
$$

The measurement points for the pressure are dependent on the measuring pressure range of the sample and are set using the Eqs. (7) to (9) [1]:

$$
\begin{aligned}
& p_{2} \approx \frac{3 p_{\min }+p_{\max }}{4}, \\
& p_{3} \approx \frac{p_{\text {min }}+p_{\max }}{2}, \\
& p_{4} \approx \frac{p_{\min }+3 p_{\max }}{4} .
\end{aligned}
$$

The measurement error $f_{C}$ in [\%] can be calculated on the basis of Eq. (10), [1]:

$$
f_{C}=\frac{C_{m}-C_{p}}{C_{p}} \cdot 100 \%,
$$

where $C_{m}$ is the correction factor for the sample, and $C_{p}$ is the real value of the correction factor that is determined on the basis of a standard measurement system, as calculated via Eq. (2). In each VCD test the standard requires the completion of a gauge check at the $8^{\text {th }}$ measuring point.

The VCD measurement error $f_{C}$ is both temperature and pressure dependent:

$$
f_{C}=f_{C}(t, p) .
$$

Fig. 4 demonstrates an example of the VCD measurement errors, measured at three different temperatures and five different pressures for each temperature.

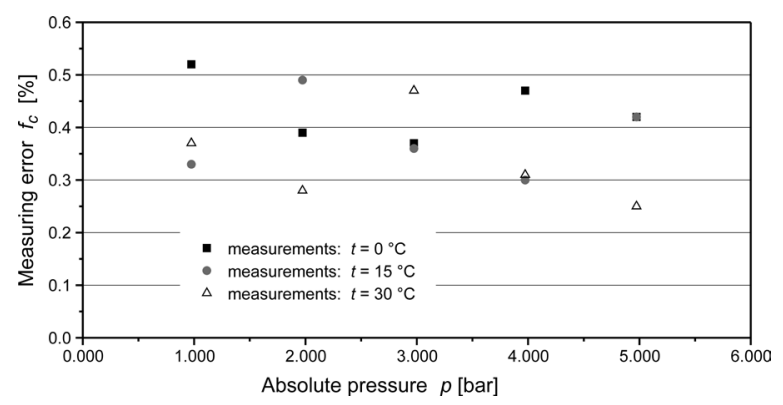

Fig. 4. Measurement errors during a VCD test 


\section{MULTI-REGRESSION MODEL FOR DETERMINING THE MEASUREMENT ERRORS IN VCD TESTS}

A regression analysis is a statistical method where the experimental data is approximated with a known regression function and/or regression model [13] to [16]. The goal of multi-regression analysis is to determine the dependency between two or more independent quantities and/or regression variables. There are many known multi-regression models, but not all of them are suitable for individual analysis. Each model has its own specific properties, advantages, and disadvantages. A multi-regression analysis also allows us to compare the influence of particular independent variables on a dependent variable. With this we can determine which variables have a larger influence and which have a lesser one. That is why, in the analysis, we may exclude variables that only negligibly influence the dependent variable.

A regression analysis is a statistical method whereby the experimental data is approximated with a known function. In general, the response of the system is determined using the following equation:

$$
y=E_{0}+\sum_{i=1}^{n} E_{i} z_{i}+\sum_{i=1}^{n} \sum_{j>i}^{n} E_{i j} z_{i} z_{j}++\sum_{i=1}^{n} E_{i i} z_{i}^{2}+\ldots
$$

where $E_{0}, E_{i}$ and $E_{i j}$ are constant coefficients of the regression estimator, $z_{i}$ is an independent variable and $y$ is a dependent variable.. The coefficients are determined using the method of least squares. For the system's response we look at the function of approximation $f_{\text {error }}$, for which we demand that it has a minimum:

$$
f_{\text {error }}=\sum_{i=1}^{n} \sum_{j=1}^{n}\left(y_{i j}-y\left(E_{0}, E_{i}, E_{i j ; j>i}, E_{i i} ; z_{i}, z_{j>i}\right)\right)^{2} .
$$

The function of the approximation error will have a minimum if the first derivative of the function $f_{\text {error }}$ with respect to the parameters $E_{i}$ is equal to 0 , but the second derivative of the function is positive. The acquired system of equations Eq. (14) is usually solved via the Gaussian method.

$$
\begin{gathered}
\frac{\partial f_{\text {error }}}{\partial E_{0}}=0=2 \sum_{i, j=1}^{n}\left(y_{i j}-y\left(E_{0}, E_{1}, z_{1}, E_{2}, z_{2}, \ldots\right)\right) \frac{\partial y}{\partial E_{0}}, \\
\frac{\partial f_{\text {error }}}{\partial E_{1}}=0=2 \sum_{i, j=1}^{n}\left(y_{i j}-y\left(E_{0}, E_{1}, z_{1}, E_{2}, z_{2}, \ldots\right)\right) \frac{\partial y}{\partial E_{1}}, \\
\frac{\partial f_{\text {error }}}{\partial E_{2}}=0=2 \sum_{i, j=1}^{n}\left(y_{i j}-y\left(E_{0}, E_{1}, z_{1}, E_{2}, z_{2}, \ldots\right)\right) \frac{\partial y}{\partial E_{2}}, \\
\vdots \\
\frac{\partial f_{\text {error }}}{\partial E_{n n}}=0=2 \sum_{i, j=1}^{n}\left(y_{i j}-y\left(E_{0}, E_{1}, z_{1}, E_{2}, z_{2}, \ldots\right)\right) \frac{\partial y}{\partial E_{n n}} .
\end{gathered}
$$

\subsection{Determining the Equations of the Regression Model with the Help of Experimental Design Theory}

The equation of the regression models can be determined on the basis of understanding the mathematical model that describes a specific physical process. However, the physical process is not always sufficiently well known, and in such an example we may help ourselves with the experimental design theory from [13] and the graphics. Within the experimental design theory there is also the perfect factor experiment, which is used when we are interested in the influence of two or more factors $(k)$ on at least two levels $(r)$, with several repetitions of the experiments under unchanged conditions $(h)$. The number of required experiments is:

$$
n=r^{k} \cdot h \text {. }
$$

If we assume that the effect of the factors is quadratic, then we need 32 experimental points. The geometrical interpretation is shown in Fig. 5, where the independent variables are marked as $z_{1}$ and $z_{2}$ :

$$
y=y\left(z_{1}, z_{2}\right)
$$

In Fig. 5, the experimental points for which we carry out the measurements are presented. The plan of the experiment $3^{2}$ also has a central point. On this basis the corresponding regression polynomial that we determine with the method of least squares is equal to:

$$
y=E_{0}+E_{1} z_{1}+E_{2} z_{2}+E_{12} z_{1} z_{2}+E_{11} z_{1}^{2}+E_{22} z_{2}^{2} .
$$

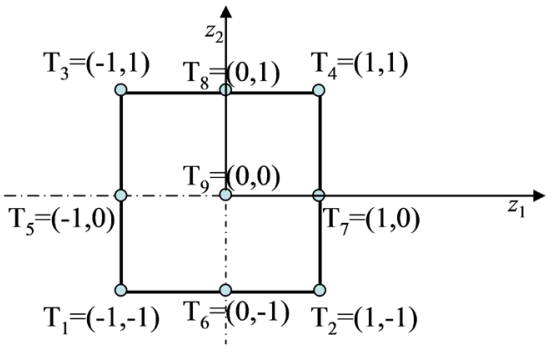

Fig. 5. The geometric interpretation of the quadratic perfect factorial experiment 32

\subsection{The use of regression analysis for estimating the VCD measurement error}

The functional dependency of the measurement error is defined with Eq. (11) $f_{C}=f_{C}(t, p)$. With a consideration of Eq. (17), we can move this dependency with a regression polynomial, Eq.(18):

$$
f_{C}=E_{0}+E_{1} t+E_{2} p+E_{12} t \cdot p+E_{11} t^{2}+E_{22} p^{2} .
$$


The main requirement of a regression analysis is that the number of available measurements is as numerous as possible. For the testing of type $1 \mathrm{VCDs}$, Fig. 4, $N=15$ measurement points were measured, thus we must determine $M=6$ parameters in the approximation equation. The measure for the estimate of suitability for the approximation is also the standard error of the estimate $S E E$, which takes into account the function of errors and the number of degrees of freedom $v=N-M$. It is defined with the expression in Eq. (19):

$$
S E E=\sqrt{\frac{f_{\text {error }}}{N-M}} .
$$

The easiest way to determine the coefficients in Eq. (18) is with the use of a regression analysis, where we consider the use of variance analysis ANOVA. The $F$ statistic in Table 3 determines whether the variation between the sample means is significant on the level 0.05 . An example of such a printout from a regression analysis on the basis of the measurements from Fig. 4 and the use of computer programs SPSS [17] or Excell are shown in Table 3.

Table 3. Regression analysis and ANOVA with the use of computer programs

\begin{tabular}{|c|c|c|c|c|c|}
\hline \multicolumn{6}{|c|}{ SUMMARY OUTPUT } \\
\hline \multicolumn{6}{|c|}{ Regression Statistics } \\
\hline \multicolumn{3}{|c|}{ Multiple $r$} & \multicolumn{3}{|c|}{0.98775} \\
\hline \multicolumn{3}{|l|}{$r^{2}$} & \multicolumn{3}{|c|}{0.97565} \\
\hline \multicolumn{3}{|c|}{ Adjusted $r^{2}$ Square } & \multicolumn{3}{|c|}{0.96212} \\
\hline \multicolumn{3}{|c|}{ SEE } & \multicolumn{3}{|c|}{0.01581} \\
\hline \multicolumn{3}{|c|}{ Observations } & \multicolumn{3}{|c|}{15} \\
\hline ANOVA & $v$ & $S S$ & $M S$ & $F$ & Significance $F$ \\
\hline Regression & 5 & 0.09008 & 0.01802 & 72.116 & $5.50981 \mathrm{E}-07$ \\
\hline Residual & 9 & 0.00225 & 0.00025 & & \\
\hline \multirow[t]{2}{*}{ Total } & 14 & 0.09233 & & & \\
\hline & & \multicolumn{2}{|c|}{ Coefficients } & & tandard Error \\
\hline \multicolumn{2}{|l|}{$E_{0}$} & \multicolumn{2}{|c|}{0.604690} & & 0.023 \\
\hline \multicolumn{2}{|l|}{$E_{1}$} & \multicolumn{2}{|c|}{-0.091278} & & 0.015 \\
\hline \multicolumn{2}{|l|}{$E_{2}$} & \multicolumn{2}{|c|}{-0.002149} & & 0.001 \\
\hline \multicolumn{2}{|l|}{$E_{12}$} & \multicolumn{2}{|c|}{0.000600} & & 0.000 \\
\hline \multicolumn{2}{|l|}{$E_{11}$} & \multicolumn{2}{|c|}{0.009292} & & 0.002 \\
\hline \multicolumn{2}{|l|}{$E_{22}$} & \multicolumn{2}{|c|}{0.000022} & & 0.000 \\
\hline
\end{tabular}

The results of the regression analysis are shown in Fig. 6. On the basis of the variance analysis, Table 3 and Fig. 6, we can conclude that the regression curve fits well with the measurements. Therefore, the significance $F$ is very small, the coefficient of multi regression $r^{2}$ is very large, as is the standard error of the estimate $S E E$, which is measured in units dependent on the variable $f_{C}$ and is very small.

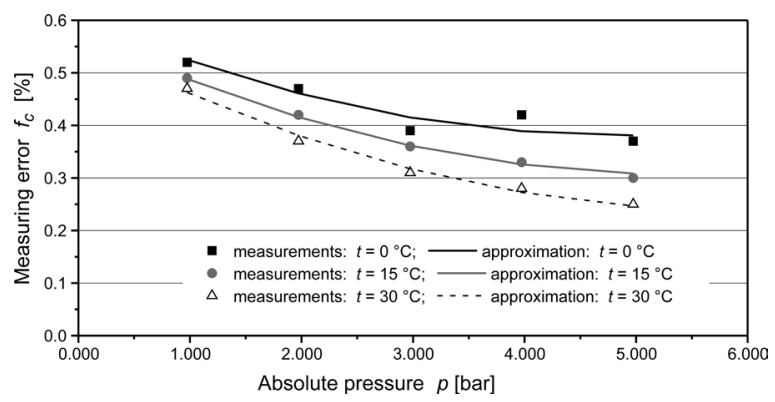

Fig. 6. A comparison between the measured values and the approximated results in accordance with Eq. (18) at parameters $r^{2}=0.976$ and $S E E=0.016 \%$

For the data from the regression analysis that are collected in Table 3 and Fig. 6, we can determine the next statistical parameters that estimate the suitability of the approximations chosen in Table 4.

Table 4. Regression analysis and ANOVA

\begin{tabular}{lc}
\hline \multicolumn{1}{c}{ Statistic } & Value \\
\hline$S E E$ & $0.0158 \%$ \\
\hline$r^{2}$ & 0.97565 \\
\hline Significance & $5.51 \cdot 10^{-7}$ \\
\hline
\end{tabular}

\section{EXPERIMENTAL ANALYSIS OF THE USE OF THE MULTI-REGRESSION MODEL FOR MEASUREMENT ERRORS DURING A VCD TEST}

For the use of a regression analysis at natural gas VDC we chose eight different models of the VDC from different manufacturers, i.e., M1 to M8. The VDCs M6, M7, and M8 met the requirements of the MID, meaning their maximum-permissible error (MPE) was $\pm 0.5 \%$. The first three models of the VCDs had an allowed error of $\pm 1 \%$. Within the selected VCDs we have also used a type 2 VDC (M4) that we calibrated as a type $1 \mathrm{VCD}$.

The sample size of the testing was 149 , namely; $30 \mathrm{M} 1,13 \mathrm{M} 2,22 \mathrm{M} 3,30 \mathrm{M} 4,20 \mathrm{M} 5,13 \mathrm{M} 6,16 \mathrm{M} 7$, and 5 M8 VCDs.

Fig. 7 shows the results of the regression analysis yields for the M7 VDC that was MID approved. Fig. 7 shows very clearly the correspondence between the measurements and the error curve. This holds for all the analysed VCDs that met the requirements of the MID. 


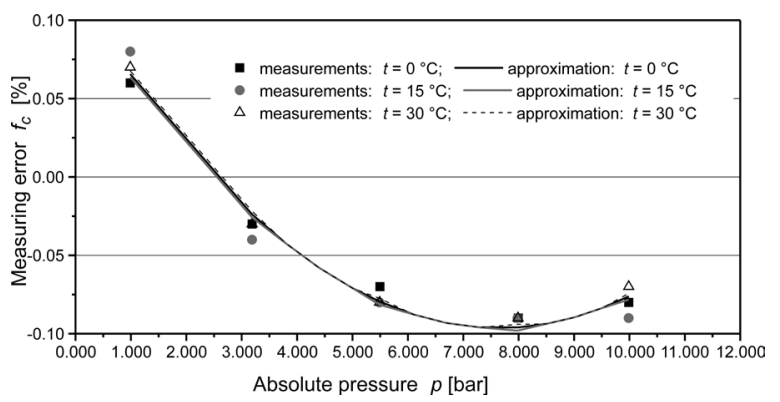

Fig. 7. Comparison between the measured and approximated data for the M7 VDC in accordance with Eq. (18) at parameters $r^{2}=0.981$ and $S E E=0.011 \%$

\subsection{Parameters of the Properties for the Regression Equations}

The most important statistical estimator for assessing the multi-regression model is the standard error of the estimate $S E E$. For the upper threshold value we took the testing facilities' measurement uncertainty of $\pm 0.035 \%$. This means that the measurement errors of the VCDs, which have $S E E$ values of $S E E= \pm 0.035$ $\%$ or less, which we calculated using a regression analysis, fit the regression model more than $90 \%$. This means that for every VCD that had its calculated $S E E$ value via a multi-regression analysis lower than $\pm 0.035 \%$, this regression model is suitable for the approximation.

The second statistical estimator with which we assessed the approximation of the regression model, is the regression coefficient $r^{2}$. It is more difficult to set the upper threshold value for the regression coefficient, because $r^{2}$ is dependent on the selected regression curve. By considering the obtained results we can set a lower threshold value for $r^{2}$ on 0.8 , which means that the measured values lie $80 \%$ on the regression curve. The VCDs with an $r^{2}$ value lower than 0.8 have a worse fit.

How well the regression model fits with the measurements determines the estimator of the significance $F$. The statistical significance $F$ is calculated within the ANOVA analysis of the variance framework. The upper threshold value for the significance estimator $F$ is 0.05 . For all the VCDs approximations whose value is under 0.05 , we can claim with a $95 \%$ probability that at least one of their independent variables has a statistical effect on a dependent variable.

In Table 5 we have a list of values for the estimators $S E E, r^{2}$, and the significance $F$ for the best and the worst VCDs that we checked during their testing. The second is the consecutive numbering of the tested VCD.

As is clear from Table 5, the $S E E$ values for the VCDs, apart from VCD M5-10, are within the selected threshold $S E E$ values for $S E E= \pm 0.1 \%$. For the VCD model M5 it turned out that no approximation of this VCD is sufficient for the required $S E E$ values. From this we can conclude that because of the specific characteristics of the VCDs M5, the chosen regression model Eq. (18) is not suitable for approximating its measured quantities. The cause of such a poor approximation is the specific metrological characteristics of the built-in pressure and temperature sensors. Here we must add that the VCDs M5 have been out of production for a long time.

The selected threshold $S E E$ value of $S E E= \pm 0.035$ $\%$ is equal to the allowed share of the measurement uncertainty of the VCD testing facilities, which is why we also studied the other suitable values.

We increased the allowed values in increments and observed how many approximated VCDs are suitable for a particular threshold value. The $S E E$ value of $S E E= \pm 0.075 \%$ is of an approximately equal order of magnitude to the measurement uncertainty of the test facility for checking the electronic VCDs $( \pm 0.167$ $\%)$. As it turns out, the results do not differ much from the $S E E$ value of $S E E= \pm 0.1 \%$. The combined measurement uncertainty of the testing facility and the approximation do not exceed $\pm 0.3 \%$, which is also the acceptable value for the measurement uncertainties of the newest flow computers.

Table 5. Values of statistical estimators of regression analysis for electronic VCDs

\begin{tabular}{ccccc}
\hline \multirow{2}{*}{$\begin{array}{c}\text { VCD } \\
\text { (corrector) }\end{array}$} & \multicolumn{3}{c}{ Statistical estimator } \\
\cline { 2 - 5 } M1 & Standard Error & $r^{2}$ & Significance $F$ \\
\cline { 2 - 5 } & M1-18 & 0.015 & 0.993 & 0.0000 \\
\hline \multirow{2}{*}{ M2 } & M2-5 & 0.098 & 0.628 & 0.0706 \\
\cline { 2 - 5 } & M2-2 & 0.031 & 0.843 & 0.0020 \\
\hline \multirow{2}{*}{ M3 } & M3-5 & 0.014 & 0.823 & 0.0000 \\
\cline { 2 - 5 } & M3-4 & 0.099 & 0.976 & 0.0000 \\
\hline \multirow{2}{*}{ M4 } & M4-12 & 0.012 & 0.839 & 0.0022 \\
\cline { 2 - 5 } & M4-21 & 0.089 & 0.311 & 0.0000 \\
\hline \multirow{2}{*}{ M5 } & M5-19 & 0.039 & 0.966 & 0.5686 \\
\cline { 2 - 5 } & M5-10 & 0.154 & 0.489 & 0.2248 \\
\hline \multirow{2}{*}{ M6 } & M6-6 & 0.018 & 0.971 & 0.0000 \\
\cline { 2 - 5 } & M6-3 & 0.044 & 0.123 & 0.9281 \\
\hline \multirow{2}{*}{ M7 } & M7-5 & 0.007 & 0.990 & 0.0000 \\
\cline { 2 - 5 } & M7-14 & 0.034 & 0.388 & 0.0000 \\
\hline \multirow{2}{*}{ M8 } & M8-5 & 0.008 & 0.968 & 0.0000 \\
\cline { 2 - 5 } & M8-1 & 0.027 & 0.774 & 0.0090 \\
\hline
\end{tabular}


The data about the number of VCDs that are approximated with Eq. (18) and meet the criteria of the $S E E$ are collected in Table 6. Besides the number, the relative shares of suitable approximations are also presented. The number of VCDs used for the approximation is indicated in the last column of the table.

From Table 6 we can see that only 4 of the 149 approximations do not comply with the SEE value of $S E E= \pm 0.1 \%$. All four approximations are of the model M5 VCDs, for which we predicted that we would need to find a better regression model.

Table 6. Threshold values of SEE and the number of suitable VCDs

\begin{tabular}{|c|c|c|c|c|c|c|}
\hline \multirow{2}{*}{\multicolumn{2}{|c|}{ SEE [\%] }} & \multicolumn{4}{|c|}{ Threshold limit } & \multirow{2}{*}{$\begin{array}{c}\text { Total } \\
\text { number }\end{array}$} \\
\hline & & $<0.035$ & $<0.05$ & $<0.075$ & $<0.1$ & \\
\hline \multirow{2}{*}{ M1 } & number & 13 & 20 & 28 & 30 & \multirow{2}{*}{30} \\
\hline & $\%$ & 43.33 & 66.67 & 93.33 & 100.00 & \\
\hline \multirow{2}{*}{ M2 } & number & 1 & 7 & 10 & 13 & \multirow{2}{*}{13} \\
\hline & $\%$ & 7.69 & 53.85 & 76.92 & 100.00 & \\
\hline \multirow{2}{*}{ M3 } & number & 13 & 20 & 21 & 22 & \multirow{2}{*}{22} \\
\hline & $\%$ & 59.09 & 90.91 & 95.45 & 100.00 & \\
\hline \multirow{2}{*}{ M4 } & number & 17 & 26 & 28 & 30 & \multirow{2}{*}{30} \\
\hline & $\%$ & 56.67 & 86.67 & 93.33 & 100.00 & \\
\hline \multirow{2}{*}{ M5 } & number & 0 & 1 & 10 & 16 & \multirow{2}{*}{20} \\
\hline & $\%$ & 0.00 & 5.00 & 50.00 & 80.00 & \\
\hline \multirow{2}{*}{ M6 } & number & 9 & 13 & 13 & 13 & \multirow{2}{*}{13} \\
\hline & $\%$ & 96.23 & 100.00 & 100.00 & 100.00 & \\
\hline \multirow{2}{*}{ M7 } & number & 16 & 16 & 16 & 16 & \multirow{2}{*}{16} \\
\hline & $\%$ & 100.00 & 100.00 & 100.00 & 100.00 & \\
\hline \multirow{2}{*}{ M8 } & number & 5 & 5 & 5 & 5 & \\
\hline & $\%$ & 100.00 & 100.00 & 100.00 & 100.00 & \\
\hline
\end{tabular}

Table 7. SEE threshold values and the number of suitable VCDs excluding M5 VCDS

\begin{tabular}{ccc}
\hline & \multicolumn{2}{c}{ SEE [\%] } \\
\cline { 2 - 3 } Limit & \multicolumn{2}{c}{ M1, M2, M3, M4, M5, M6, M7, M8 } \\
\cline { 2 - 3 } & Total number & \% \\
\hline$<0.035$ & 74 & 57.36 \\
\hline$<0.050$ & 107 & 82.95 \\
\hline$<0.075$ & 121 & 93.80 \\
\hline$<0.100$ & 129 & 100.00 \\
\hline Total & 129 & 100,00 \\
\hline
\end{tabular}

If we exclude all the model M5 VCDs from the analysis, then we only take into account 129 VCDs. The results are listed in Table 7. From these results collected in Tables 6 and 7 we can see that the VCDs with MID approval meet the SEE threshold already at $0.05 \%$. These results confirm our hypothesis, that it is possible to approximate the measurements of naturalgas VCDs sufficiently well with the multi-regression model in Eq. (18). The exactness of the approximation is dependent on the allowed threshold SEE values. Based on the results of the multi-regression analysis we can claim that the regression model only poorly approximates the measurements from the VCDs of model M5, and we may, as a consequence, exclude them from our analysis. However, it must be understood that our research did not demonstrate the model M5 VCD to be unfit for use, but because of its uncertainties our selected regression model cannot describe it well enough.

\subsection{Reducing the Numbers of Measurement Points}

The optimum choice of measurement points for the VCDs testing in the field could be determined on the basis of [18] and [19]. The number of measurements points was obtained with the aim of reducing the calibration curve's uncertainty. The authors in [19] present the design of a calibration using experimental design techniques. The optimum calibration plan for the measurement chain is identified by suitably elaborating the error-propagation law suggested by the ISO Guide [20]. In our case we want to keep the valid methodology and national procedure during the testing procedure in our laboratory. Our findings were that the suggested regression Eq. (18) fits our measurements exceptionally well, especially for the MID-approved VCDs that are being produced recently. Based on these findings it stands to reason that we need only 9 measurement points and the regression analysis. The number of selected measurement points without repetition comes from the experimental design theory, Fig. 5. A reduced number of measurement points is proposed for the testing of the VCDs in the field. From this we propose the following reduced designated testing points, as depicted in Table 8.

Table 8. The designated testing measurement points for 9 measuring points

\begin{tabular}{cccc}
\hline Temperature $\left[{ }^{\circ} \mathrm{C}\right]$ & $p_{1 \min }$ & $p_{3}$ & $p_{5 \max }$ \\
\hline$t_{1}$ & $1 \rightarrow$ & $2 \rightarrow$ & $3 \downarrow$ \\
\hline$t_{2}$ & $6 \downarrow$ & $5 \leftarrow$ & $4 \leftarrow$ \\
\hline$t_{3}$ & $7 \rightarrow$ & $8 \rightarrow$ & $\rightarrow 9$ \\
\hline & & & \\
\hline Measuring point, Fig. 5 & $z_{1 \min }$ & $z_{10}$ & $z_{1 \max }$ \\
\hline$z_{1 \min }$ & $T_{1}$ & $T_{5}$ & $T_{3}$ \\
\hline$z_{10}$ & $T_{6}$ & $T_{9}$ & $T_{8}$ \\
\hline$z_{1 \max }$ & $T_{2}$ & $T_{7}$ & $T_{4}$ \\
\hline
\end{tabular}

For the example in Fig. 7 we have made an approximation with only 9 measurement points. The 
Table 9. The comparison of SEE values considering 9 or 15 measurement points

\begin{tabular}{ccccccc}
\hline$f_{C}$ & $p$ & $t$ & \multicolumn{2}{c}{ Approximation: 9 points } & \multicolumn{2}{c}{ Approximation: 15 points } \\
\cline { 4 - 7 }$[\%]$ & {$[\mathrm{bar}]$} & {$\left[{ }^{\circ}\right]$} & $f_{C, a p r}$ & $f_{C, a p r}-f_{C}[\%]$ & $f_{C, a p r}$ & $f_{C, a p r}-f_{C}[\%]$ \\
\hline 0.06 & 0.98749 & -0.01 & 0.069 & 0.009 & 0.066 & 0.006 \\
\hline-0.07 & 5.49396 & 0.00 & -0.078 & -0.008 & -0.080 & -0.010 \\
\hline-0.08 & 9.98705 & -0.01 & -0.081 & -0.001 & -0.076 & 0.004 \\
\hline 0.08 & 0.98749 & 15.02 & 0.069 & -0.011 & 0.064 & -0.016 \\
\hline-0.08 & 5.49396 & 15.00 & -0.078 & 0.002 & -0.082 & -0.002 \\
\hline-0.09 & 9.98705 & 15.00 & -0.081 & 0.009 & -0.078 & 0.012 \\
\hline 0.07 & 0.98749 & 30.00 & 0.072 & 0.002 & 0.068 & -0.002 \\
\hline-0.08 & 5.49396 & 30.00 & -0.074 & 0.006 & -0.078 & 0.002 \\
\hline-0.07 & 9.98705 & 30.00 & -0.078 & -0.008 & -0.074 & -0.004 \\
\hline
\end{tabular}

difference in the $S E E$ values, if we have approximated it with 9 points, or if we have calculated the coefficients of the estimator on 15 points, is less than $0.005 \%$. The data for the comparison of regressions is collected in Table 9.

\subsection{The Combined Estimate of the Measuring Uncertainty with a Consideration of the Regression Analysis}

For our estimate of the measurement uncertainty we followed the GUM [20] and [21]. The measurement uncertainty of the measurement errors $U\left(f_{C}\right)$ is dependent on the measurement uncertainty of the testing facility for the control of VCDs $U\left(f_{C}\right)_{m s}$, which accounts for $1 / 3$ of the measurement error, which is $0.167 \%$, and of the measurement uncertainty of the approximation of the measurement errors $U\left(f_{C}\right)_{a p r}$.

$$
U\left(f_{C}\right)=\sqrt{U\left(f_{C}\right)_{m s}^{2}+U\left(f_{C}\right)_{a p r}^{2}} .
$$

If we take into account the $S E E$ because of the approximation, then the estimate of the measurement uncertainty for the correlation factor equals:

$$
U\left(f_{C}\right)=\sqrt{(1 / 3 M P E)^{2}+(2 \cdot S E E)^{2}} .
$$

Taking in consideration our analysis, we can estimate the combined measurement uncertainty of the correction factor on the basis of Eq. (21), with the results collected in Table 10.

Based on our estimate (Table 10) our combined measurement uncertainty is less than $\pm 0.3 \%$, which is for all VCDs lower than the acceptable measurement errors. Modern VCDs must allow the entry of regression equation coefficients, so that the combined measurement uncertainty for standard or norm naturalgas volumes is less than $\pm 0.5 \%$.
Table 10. The combined measurement uncertainty of the VCDs' measurement error

\begin{tabular}{cccc}
\hline $\operatorname{SEE}\left(f_{C}\right)$ & $1 / 3 \mathrm{MPE}$ & $2 \mathrm{SEE}$ & $U\left(f_{C}\right)$ \\
\hline $\mathrm{SEE}= \pm 0.000 \%$ & \pm 0.167 & \pm 0.000 & \pm 0.167 \\
\hline $\mathrm{SEE}= \pm 0.035 \%$ & \pm 0.167 & \pm 0.070 & \pm 0.181 \\
\hline $\mathrm{SEE}= \pm 0.070 \%$ & \pm 0.167 & \pm 0.140 & \pm 0.218 \\
\hline $\mathrm{SEE}= \pm 0.100 \%$ & \pm 0.167 & \pm 0.200 & \pm 0.260 \\
\hline
\end{tabular}

Modern flow meters enable the measurement of natural-gas volumes to better than $\pm 0.5 \%$. An example of this would be some ultrasonic flow meters, which can reach measurement uncertainties values of less than $\pm 0.2 \%$. With the proposed measurement method it is possible to reduce the measurement uncertainty of the VCDs to less than $\pm 0.3 \%$, which would enable a new generation of VCDs.

\section{CONCLUSIONS}

Based on the multi-regression analysis results we were able to come to the following conclusions:

- The most important statistical estimator for estimating the suitability of a multi-regression analysis used for any natural-gas VCD is the standard error of the estimate SEE. Additional important statistical parameters are the regression coefficient $r^{2}$ and the significance $F$.

- The proposed regression model is suitable for use with the control of all VCDs, especially modern MID-approved VCDs. However, the estimator is not suitable for the M5 VCDs that are out of production, because of the specifics of the sensors.

- The VCD approximation suitability on the basis of the chosen regression model differs depending on the model of the VCD. Of all the tested VCDs, 
the regression model approximates VCD M7 with the best fit.

- The regression model approximates the VCD measurements by considering the demanded $S E E$ threshold value of $S E E= \pm 0.035 \%$ in $57.4 \%$ of cases. And for an $S E E$ threshold value of $S E E=$ $\pm 0.075 \%$, which approximately corresponds to the measuring uncertainty of the testing facility $( \pm 0.167 \%)$, it suitably approximates for more than $93 \%$ of the VCDs.

- The proposed method of measurement represents an upgrade to the national verification procedures for the testing of VCDs.

- In the case that we perform a multi-regression analysis of the VCD test on the basis of the reduced number of measurement points ( 9 points), and the results conform to the requirements, then the probability of an error is less than $25 \%$. In the case of model M4 the probability of an error was $13.3 \%$, so we may claim with a certainty of $86.7 \%$ that the results will also conform to a 15-point test. Meanwhile, for VCDs M6, M7, and M8, the prediction is $100 \%$. A reduced number of measurement points is well suited to the field testing of VCDs.

- In a comparison of the regression analysis based on 15 or 9 points, in most cases the $S S E$ values will increase, as will the values of the $r^{2}$ estimator, but the values of the significance $F$ will, however, decrease in most cases.

- Modern VCDs generally allow the entry of the regression-estimator constants from Eq. (18).

- A regression analysis is especially valuable for VCD testing, because it can shorten the testing times.

- We have demonstrated that we may use a regression analysis based on 9 points instead of 15 , which is the keystone of field measurement procedures.

- The proposed testing procedure can be automated.

\section{REFERENCES}

[1] EN 12405-1:2005+A2:2010. Gas meters - Conversion devices - Part 1: Volume conversion. European Committee for Standardization, Brussels.

[2] PTB - Prüfregeln: Band 20:1993. Elektronische Mangenumwerter für Gas. PTB, Braunschweig, Berlin.

[3] Rules on Measurement Instruments (Pravilnik o spremembah in dopolnitvah pravilnika o merilnih instrumentih) (2006). Official Gazette of the Republic of Slovenia, nr. 42/2006. (in Slovene)
[4] L135 (2004), L137 (2009). Directive 2004/22/EC of the European Parliament and of the Council of 31 March 2004 on measuring instruments. vol. 47, p. 1-80.

[5] Oprešnik, M. (1992). Thermodynamics, University of Ljubljana, Faculty of Mechanical Engineering, 4th ed., Ljubljana (in Slovene).

[6] EN 12213-1,2,3:2009. Natural gas - Calculation of compression factor. European Committee for Standardization. Brussels.

[7] Buonanno, G., Carotenuto, A., Dell'Isola, M. (1998). The influence of reference condition correction on natural gas flow measurement. Measurement, vol. 23, p. 77-91, Dol:10.1016/ s0263-2241(98)00009-8.

[8] Ficco, G., Dell'Isola, M., Vigo, P., Celenza, L. (2015). Uncertainty analysis of energy measurements in natural gas transmission networks. Flow Measurement and Instrumentation, vol. 42, p. 58-68, D0I:10.1016/j.flowmeasinst.2015.01.006.

[9] Arpino, F., Dell'Isola, M., Ficco, G., Vigo, P. (2014). Unaccounted for gas in natural gas transmission networks: Prediction model and analysis of the solutions. Journal of Natural Gas Science and Engineering, vol. 17, p. 58-70, D0l:10.1016/j. jngse.2014.01.003.

[10] Kramer, R., Mickan, B. (2009). Gas Measurement and Gas Meters Testing in Practise. PTB-Mitteilungen, vol. 119, no. 1, p. 16-22.

[11] Blagojevič, B., Cimerman, F. (2013). Knowledge of natural gas chemical composition in Plinovodi d.o.o. transmission system. ZSIS, Ljubljana. (in Slovene)

[12] PTB - Prüfregeln: Band 30:2003. Messgeräte für Gas, Hochdruckprüfung von Gaszählern. PTB, Braunschweig und Berlin.

[13] Montgomery, D.C. (2013). Design and Analysis of Experiments. $8^{\text {th }}$ Ed., John Wiley \& Sons, Inc., Hoboken.

[14] Širok, B., Blagojević, B., Bullen, P. (2008). Mineral Wool Production and properties. Woodhead Publishing Limited, Cambridge, Dol:10.1533/9781845694456.

[15] Spiegel, M., Schiller, J., Srinivasan, A. (2013). Schaum's Probability and Statistics, $4^{\text {th }}$ Ed., McGraw-Hill, New York.

[16] Grabec, I., Gradišek, J. (2000). Description of the Random Phenomena. University of Ljubljana, Faculty of Mechanical Engineering, Ljubljana. (in Slovene)

[17] IBM Software: SPSS Statistic Desktop version 22.0 for Windows.

[18] Betta, G., Dell'Isola, M. (1996). Optimum choise of measurement points for sensor calibration. Measurement, vol 17., no. 2, p. 115-125, D0l:10.1016/0263-2241(96)00019-X.

[19] Betta, G., Dell'Isola, M., Frattolillo, A. (2001). Experimental design techniques for optimising measurement chain calibration. Measurement, vol 30, p. 115-127, D0l:10.1016/ S0263-2241(00)00060-9.

[20] ISO/IEC Guide 98-3:2008. Uncertainty of measurement - Part 3. Guide to the expression of uncertainty in measurement (GUM: 1995). International Organization for Standardization Geneva.

[21] Adunka, F. (2007). Messunsicherheiten: Theorie und Praxis. Auflage 3, Vulkan-Verlag, Essen. 